\title{
Junctional communication of embryonic cells after induction
}

\author{
Zeng Mibai (Tseng Mipai) and Jiang Wansu \\ shanghai Institute of Cell Biology, Academia Sinica
}

\begin{abstract}
Cell couplings before and after neural induction in embryos of Cynops orientalis were studied by means of single cell injection of Lucifer Yellow. Differences both in incidence and the extent of cell couplings were demonstrated. Results of cell couplings were correlated with electron microscopic observations of freeze-etching replicas.
\end{abstract}

Key Words:cell communication, gap junction, neural induction.

\section{INTRODUCTION}

It has been reported that by electron microscopic observations of freeze-etching replicas, connexon configurations of gap junctions of cells before and after neural induction showed a quite evident difference. Before induction, the connexons were arranged more tightly in a more or less regular array, while those thereafter were arranged loosely and more irregularly[1]. Morphological changes in connexon configurations were found to be closely correlated with coupling states [2-6], but there are works contradictory to this view[7]. Most of these studies were carried out with a variety of treatments that modify intracellular homeostasis[2-7]. Observations of naturally occurring in situ process are no less important in verifying the relationship of these two phenomena.

Dye coupling is generally considered as one of the assays to detect intercellular transference of molecules smaller than 1000-1500 dalton via gap junctions. Initially, dyes such as fluorescein were used to demonstrate cell coupling, but they passed through non-junctional membrane to some extent. Later, Lucifer Yellow CH, a highly fluorescent sulphonated naphthelimide dye with molecular weight of 457.3 was introduced[8]. Because of its being non-toxic, spreading rapidly and unable to cross non-junctional membrane, it has been proved to be a very useful marker to study junctional communication for adult cells as well as for cells in embryonic development[ 9- 14].

Since neural induction is one of the important developmental processes, cell 
coupling before and after induction were studied by means of single cell injection of Lucifer Yellow. Differences both in incidence and extent of cell couplings were demonstrated. Results of cell couplings were supplemented freeze-etching replica observations.

\section{MATERIAL AND METHODS Embryological manipulation}

Embryos of Cynops orientalis, a species of Chinese newt, were used. Naturally laid eggs were collected and reared at $20 \pm 1^{\circ} \mathrm{C}$. As shown in the accompanying diagram (Fig. 1), for
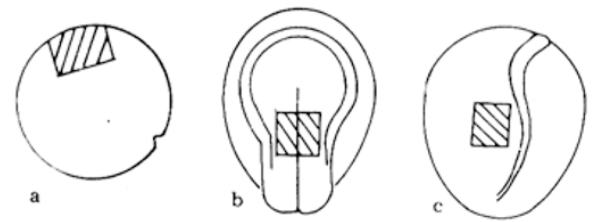

Fig. 1 Diagram showing embryological manipulation, a) early gastrula, b) mid-neurula. Shaded area indicates the explant taken for freeze-etching preparation and the site where a single cell injection was made. c) mid-neurula, shaded area shows lateral epithelium where a single cell injection was made.

series 1 , before induction, ectoderm of early gastrula was chosen when the dorsal lip first makes its appearance. Lucifer Yellow was injected into one cell of blastocoel roof. For freezeetching preparation, an ectoderm piece was taken from the blastocoel roof.

For series 2, after induction, mid-neurula stage was used when neural plate has been clearly demarkated by the rising neural fold. Lucifer Yellow was injected into one neuro-epithelium cell of trunk neural plate. For freeze-etching preparation, trunk neural plate was extirpated not including neural fold.

For series 3, to compare with neuro-epithelium cells, one cell from lateral epithelium of the same stage as series 2 was also injected.

\section{Lucifer Yellow injection}

A $2 \%$ aqueous solution of Lucifer Yellow $\mathrm{CH}$ (lithium salt, Sigma) was pressure injected by an Eppendorf Microinjector 5242. Our experimental equipment did not offer simultaneous injection and fluorescence microscopy, and it took 2-3 minutes to transfer the injected embryo to fluorescence microscope. Cell-to-cell spread of fluorescence was examined immediately and 20-30 minutes later with epi--illumination under an Opton microscope using HBO 50 Mercury lamp with standard excitation filters. Micrugraphs were taken with Kodak Tri-X pan film 400 ASA.

\section{Freeze-etching preparation}

All embryological extirpates were fixed at $4^{\circ} \mathrm{C}$ with $2.5 \%$ glutaraldehyde in $0.1 M$ cacodylate buffer at $\mathrm{pH} 7.4$, rinsed with buffer, soaked with $30 \%$ glycerine for at least 2 hours. The material was rapidly frozen in liquid nitrogen, fractured and etched in a Baltzer BAF400D. The replicas were observed with Zeiss EM109. 


\section{OBSERVATION AND DISCUSSION}

Dye transfer usually appeared 1-2 minutes after injection. Both transfer and nontransfer samples were examined again 20-30 minutes later to insure the extent of dye spread. In order to exclude the passage of dye through cytoplasmic bridge persisting between cleaving sister cells, only those samples of injected cell connected with at least two fluorescent neighbors were counted as "transfers", and "nontransfers" were those with only the injected cell showing fluorescence.

Incidence of dye coupling of the three series were summarized in Table 1.

Table 1. Incidence of dye coupling of embryonic cells before and after induction

\begin{tabular}{clccc}
\hline Series & Embryonic cells & $\begin{array}{c}\text { Number of } \\
\text { cells injected }\end{array}$ & transfers & non-transfers \\
\hline 1 & $\begin{array}{l}\text { Ectoderm cells } \\
\text { of early gestrula }\end{array}$ & 49 & $15(31 \%)$ & $34(69 \%)$ \\
\hline 2 & $\begin{array}{l}\text { Neuroepithelium } \\
\text { cells of mid-neurula }\end{array}$ & 42 & $36(86 \%)$ & $61(14 \%)$ \\
\hline 3 & Lateral epithelium & & & \\
\hline
\end{tabular}

Differences in dye coupling of the first two series were evidently shown. For series 1 , ectoderm cells of early gastrula, most of the cases (69\%) are non-transfers (Fig. 2a) and dye transfer occurred only in $31 \%, 15$ out of 49 injections (Fig. $2 \mathrm{~b}$ ). For serise 2, neuro-epithelium cells of mid-neurula, majority of the cases (86\%),36 out of 42 injecitons, displayed dye coupling. Concomitantly with the differences in dye coupling incidence, the extent of dye spread of these two series was also differed. For ectoderm cells, dye spread usually did not exceed three cells, including the injected one (Fig.2b). In neuro-epithelium cells, without any exception, dye spread to more than three cells usually forming a fluorescent zone around the injected cell(Figs.3a, b).

For the first series, ectoderm cells of early gastrula were taken from the stage before the commencement of neural induction, and the cells have not yet come into contact with the inducing mesoderm. And for the second series, when the neuroepithelium cells were taken from the stage after completion of neural induction, and the cells have already been induced to develope into neural epithelium.

From results of dye coupling, it seems that cells before and after neural induction differ both in incidence and extent of dye coupling. But it may be questioned whether the difference in cell coupling solely reflects the result of induction and 
dosen't represent the age difference of the two stages used, as mid neurula is older than early gastrula by 47 hours under our experimental condition $\left(20 \pm 1^{\circ} \mathrm{C}\right)$. In order to answer this question, the third series of experiment was carried out using lateral epithelium cells of the same age from which neuro-epithelium cells were taken. These cells were of the same embryological age as those of series 2 , and they were not underlain by inductive mesoderm and were not the products of induction. Results of Lucifer Yellow injections showed that both incidence and the extent of dye coupling of cells of series 3 are different from those of series 2 , but are similar to those of series 1 (Table 1, Fig. 4). Aging of embryonic cells
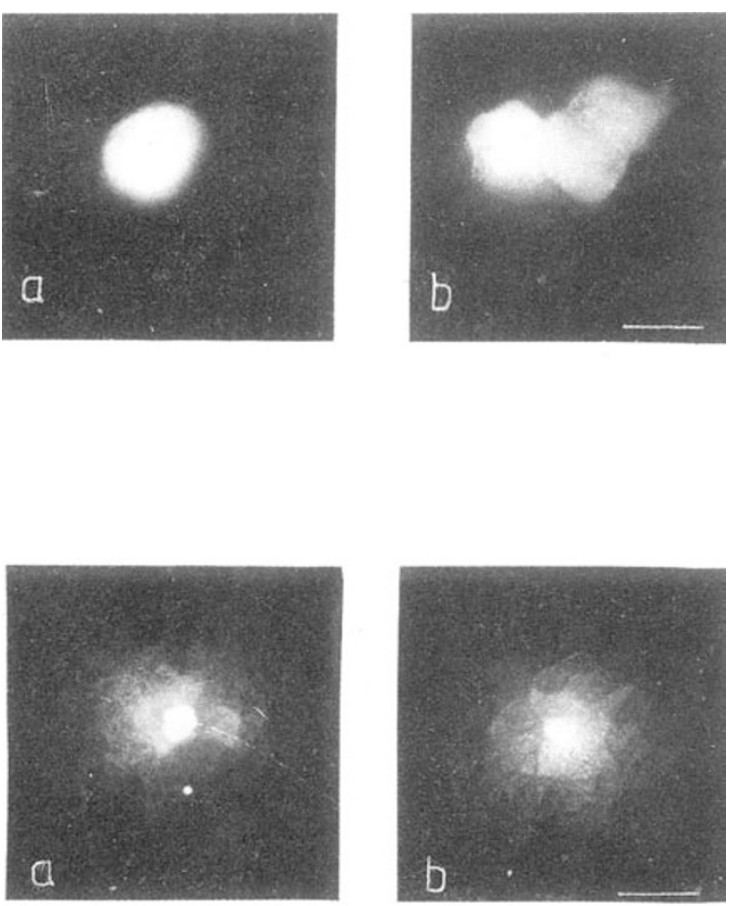

Fig. 3 Lucifer Yellow was injected into a single neuro-epithelium cell of mid-neurula. Dye spread to its neighbouring cells forming a fluorescent zone. Scale bar $=50 \mu \mathrm{m}$.

Fig. 4 Lucifer Yellow was injected into a single cell of the lateral epithelium cell of mid-neu rula. a) The dye remained in the injected cell. b) The dye was injected into the cell on the left and it was transferred to two other cells. The cell farther away from the injected Cell showed very faint fluorescence.

Scale bar $=50 \mu \mathrm{m}$. 
did not change cell coupling (compare series 1 and series 3 in Table 1), but neural induction did elicit such a change(compare series 1 and series 2 in Table 1). It is now reasonable to conclude that dye coupling of embryonic cells differed before and after neural induction. After neural induction not only the incidence of dye coupling was increased but also the extent enlarged.

This difference in dye coupling was well correlated with morphological finding from electron microscopic observations of freeze-etching replicas. Cells before and after neural induction differed in arrangements of connexons as indicated by their regularity and packing density[1]. Furthermore, freeze-etching replica observations carried out simultaneously with dye injection experiment indicated an obvious difference in frequency of occurrence and size of gap junctions.

For each of the first two series, careful searching for gap junctions were carried out for more than 20 grids from 10 samples. Only 6 gap junctions were found in the series of ectoderm cells of early gastrula and 39 gap junctions were recorded in the series of neuro-epithelium cells. In most of ectoderm cells scarcely any gap junctions could be found even in very large area of fractured membrane, but for the neuro-epithelium cells, the searching work was much easier, sometimes more than two gap junctions might be detected within a small area. Although strict quantitative comparisons were lacking, yet the difference in the frequency of gap junction occurrence remained obvious. Thus, this will provide structural basis for higher incidence and larger extent of cell coupling in series 2.

Sizes of gap junctions were shown in Table 2. The gap junctions observed on freeze-etching replicas were sometimes not complete due to the chance in fracturing, and incomplete ones were usually the larger ones of the group. In measurement of gap junctions area of the two series, only the complete ones were included (Table 2). For ectoderm cells, all the 6 gap junctions found were complete and their areas measured. 3 were below $0.01 \mu \mathrm{m}^{2}$, and the largest one was only 0.09 $\mu \mathrm{m}^{2}$ (Fig. 5). For the neuro-epithelium cells, among the 39 gap junctions, 19 were incompletes and most of them appeared to be of large sizes. Of the 20 measured, 11 were between $0.01 \mu \mathrm{m}^{2}-0.10 \mu \mathrm{m}^{2}$ with 6 below $0.01 \mu \mathrm{m}^{2}$ and 3 above $0.10 \mu \mathrm{m}^{2}$ the largest one reached $0.80 \mu \mathrm{m}^{2}$ (Fig. 6).

Table 2. Sizes of gap junctions in embryonic cells before and after induction

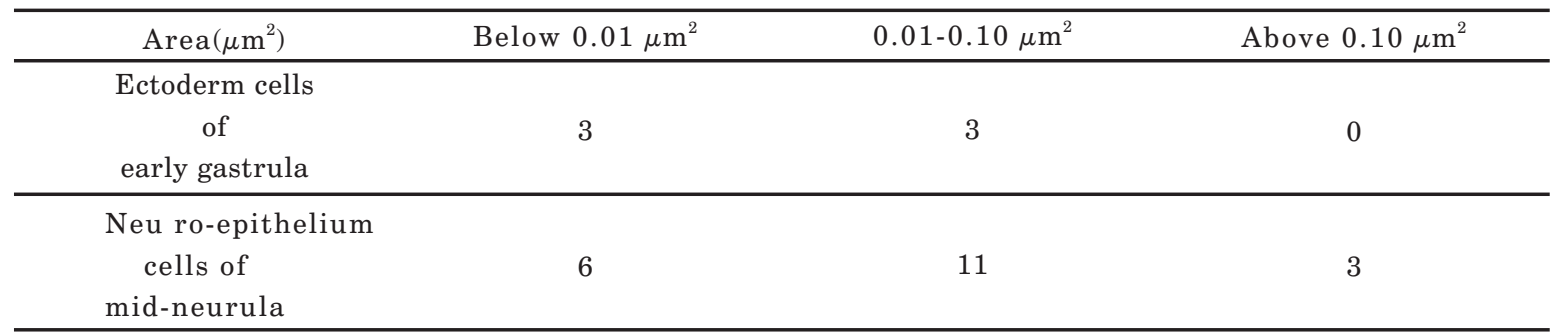


In embryonic cells, gap junctions were observed to be of dynamic nature and their sizes vary from minute aggregates to large maculae, and these were usually taken as steps in the process of assembly of gap junctions[15-18]. Similar observations were described in regenerating liver[19] and in reaggregating hepatoma cells[20]. In our observations, the range of areas among junctions of a series was observed very wide too. Average area of each series could hardly reflect the size distribution of that series. Therefore, in comparing the size distribution, the area of the largest gap junction of each of these two series should be taken into consideration. The tendency that gap junction of neuro-epithelium cells to become larger is of significance.

The increase of junctional communication of cells in neural plate, in comparison with ectoderm cells and with lateral epithelium cells, is no doubt a consequence of neural induction. When ectoderm cells once become induced, cell differentiation follows.

It is very probable that at the beginning of neural differentiation, more junctional communication is required for transfer of information and for stabilization of acquired differentiation state.

In contrast to neural plate cells, epithelium cells are determined at a much earlier stage of development. Ectoderm isolated from animal half of mid-blastula embryo developed into epithelium cells[21]. Thus, stage at which epithelium cell was examined for cell coupling was long after its determination. In comparison with neural plate cells of the same stage, these cells were in a much later stage of differentiation. And, though intercellular communication is still indispensable, it might be supposed that the role may be different from those of neural plate cells which were at the very beginning of differentiation. There is evidence showing that after embryonic induction, intercellular communication via gap junctions plays an important role in further differentiation of the inducted cells[22]. The increased activity of cell coupling of the neuro-epithelium cells offer another evidence. Up

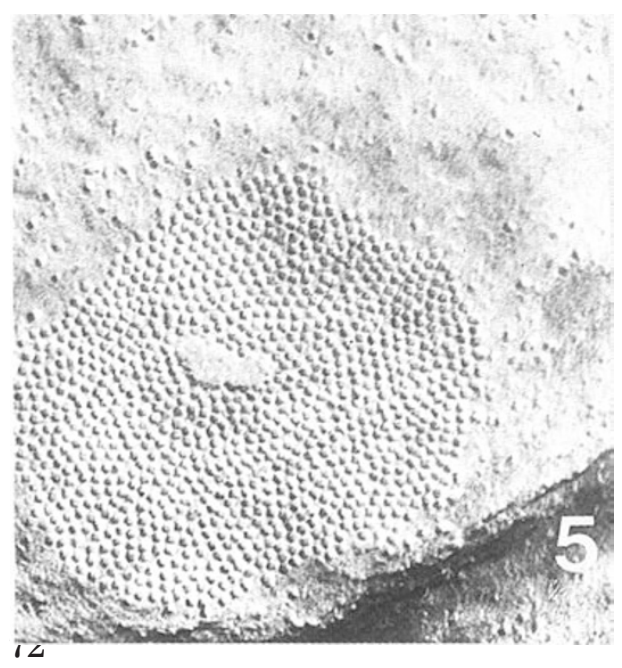

Fig. 5 Freeze-etching view of an ectoderm cell of early gastrula, showing the largest gap junction of the group, with an area of $0.09 \mu \mathrm{m}^{2}$.

Scale bar $=0.01 \mu \mathrm{m}$ 


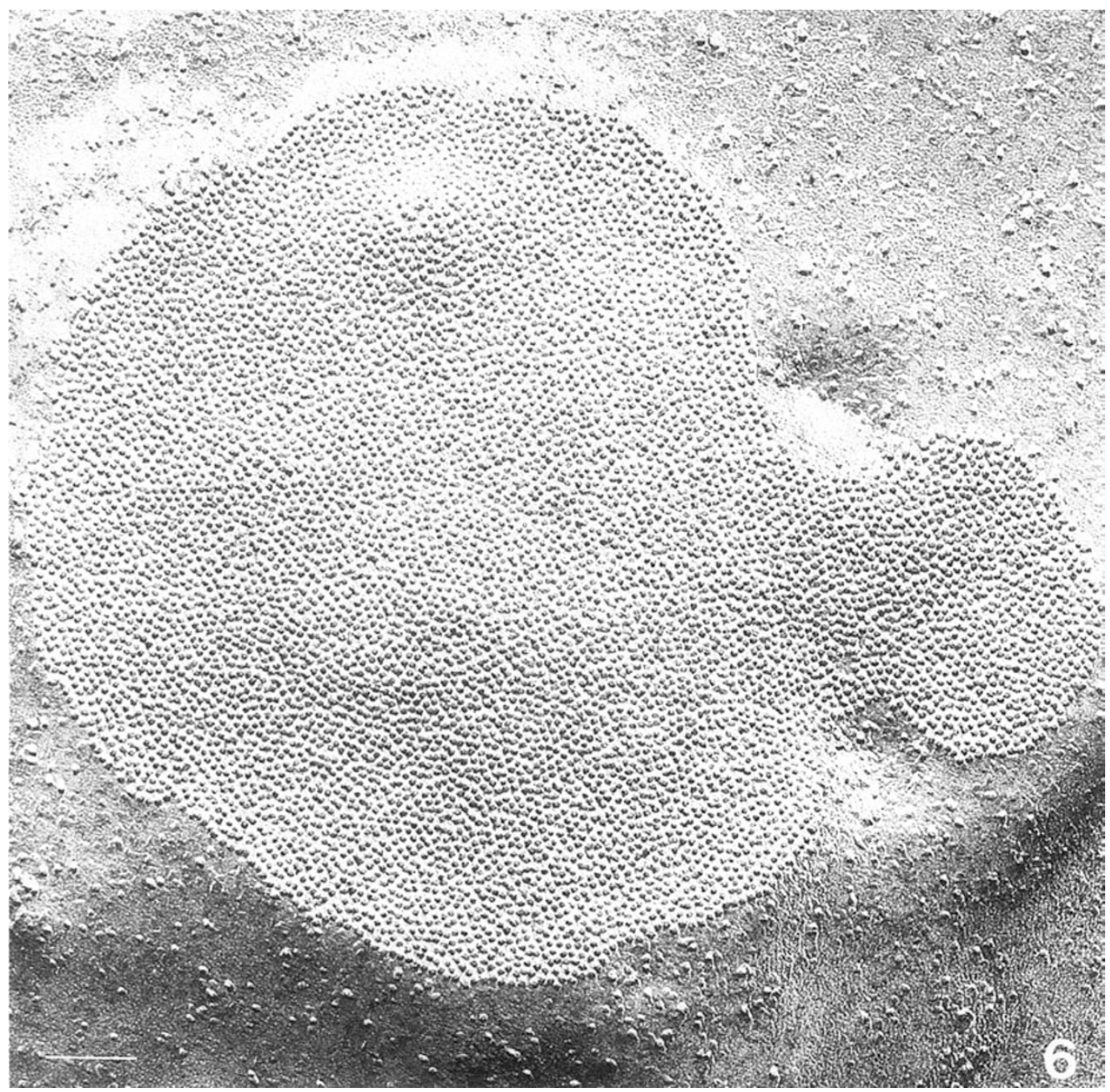

Fig. 6 Freeze-etching view of a neuro-epithelium cell of mid-neurula. This is the largest gap junction of the 20 measured, and its area is $0.80 \mu \mathrm{m}^{2}$.

Scale bar $=0.01 \mu \mathrm{m}$.

to now, only very limited number of developing cells have been assessed for both morphological and functional changes in cell communication. For better understanding, more data should be accumulated, and the role of intercellular informa tion transfer should be explored.

\section{ACKNOWLEDGMENT:}

Project supported by the Science Fund of the Academia Sinica.

\section{REFERENCES}

[ 1 ] Tseng MP, Zhou MY. Gap junctions of embryonic cells after induction. Acta Biol Exp 
Junctional communication of embryonic cells after induction

Sinica 1986; 17: 219-243.

[2] Peracchia C, Dulhunty AF. Low resistance junction in crayfish. Structural changes with functional uncoupling. J Cell Biol 1976; 70: 419-439.

[3] Baldwin KM. Cardiac gap junction configuration after an uncoupling treatment as a function of time. J Cell Biol 1979; 82: 66-75.

[4] Peracchia C. Structural correlates of gap junction permeation. Inter Rev Cytol 1980; 66: 81-146.

[5] Peracchia C, Peracchia L. Gap junction dynamic, reversible effects of hydrogen ions. J Cell Biol 1981; 87: 719-723.

[6] Chuang-Tseng MP, Chuang HH, Sandri C, Akert K. Gap Junctions and impulse propagation in embryonic epithelium of amphibia. Cell Tissue Res 1982; 225: 249-258.

[7] Hanna RB, Ornberg RL, Reese TS. Structural details of rapidly frozen gap junctions. In: Bennett NVL, Spray DC, eds. "Gap Junctions". Cold Spring Harbor Laboratory, 1985 : 23-32.

[8] Stewart WW. Functional connections between cells as revealed by dye-coupling with a highly fluorescent naphthalimide tracer. Cell 1978; 14:741-759.

[9] Sheridan JD. Dye movement and low resistance junctions between reaggregated embryonic cells. Dev Biol 1971; 26: 627-636.

[10] Lo CW, Gilula NB. Gap junctional communication in preimplantation mouse embryo. Cell $1979 ; 18: 399-410$.

[11] Lo CW, Gilula NB. Gap junctional communication in the post-implantation mouse embryo Cell 1979; 18:411-422.

[12] de Laat SW, Tertoolan LGJ, Dorresteigh AWC, van den Biggelaar JAM. Intercellular communication pattern are involved in cell determination in early molluscan development. Nature 1980; 287: 546-548.

[13] Warner AE, Lawrence PA. Permeability of gap junctions at the segment border in insect epidermis. Cell 1982; 28: 243-252.

[14] Weir MP, Lo CW. Gap-junctional communication compartments in the Drosophila wing imaginal disk. Dev Biol 1984; 102: 130-146.

[15] Revel JP, Yip P, Chang LL. Cell function in the early chick embryo in a freeze etch study. Dev Biol 1973; 35:302-317.

[16] Decker Rg, Friend DS. Assembly of gap junctions during amphibian neurulation. J Cell Biol 1974; 62: 32-47.

[17] Dasgupta JD, Eyed NS. Spatio-temporal distribution of gap junctions in Zebra fish embryo. Wilhelm Roux's Archiv Entwicklungsmech Org $1982 ; 191$ : 378-380.

[18] Tseng MP, Wang XM. Cell junction and neural tube formation in Cynops orientalis. Acta Biol Exp Sinica 1984; 17: 219-243.

[19] Yee AG, Revel JP. Loss and reappearance of gap junctions in regenerating liver. J Cell Biol $1978 ; 78$ : 554-564.

[20] Johnson R, Hammer M, Sheridan JD, Revel JP. Gap junction formation between reaggregated Novikoff Hepatoma cells. Proc Nat Acad Sci USA 1974; 71: 4536-4540. 
[21] Nieuwkoop PD. The formation of the mesoderm in urodelean amphibian. I. Induction of mesoderm. Wilhelm Roux's Archiv Entwicklungsmech Org 1969; 162: 341-373.

[22] Zeng MB (Tseng MP), Chert YH. The effect of antibodies against the gap junction protein on differentiation of induced embryonic cells. Scientia Sinica 1988; 31:13151319

Received 26-8-1988.

Accepted 7-1-1989. 